\title{
ON THE BERGMAN KERNEL AND BIHOLOMORPHIC MAPPINGS OF PSEUDOCONVEX DOMAINS
}

\author{
BY CHARLES FEFFERMAN
}

Communicated by E. M. Stein, October 30, 1973

THEOREM 1. Let $D_{1}, D_{2} \subset C^{n}$ be strictly pseudoconvex domains with smooth boundaries and suppose that $F: D_{1} \rightarrow D_{2}$ is biholomorphic (i.e., $F$ is an analytic homeomorphism). Then $F$ extends to a diffeomorphism of the closures, $\bar{F}: \bar{D}_{1} \rightarrow \bar{D}_{2}$.

The main idea in proving Theorem 1 is to study the boundary behavior of geodesics in the Bergman metrics (see [2]) of $D_{1}$ and $D_{2}$. To do so, we use a rather explicit formula for the Bergman kernels of $D_{1}$ and $D_{2}$. We begin with a few definitions. Let $D=\left\{z \in C^{n} \mid \psi(z)>0\right\}$ be a strictly pseudoconvex domain, where $\psi \in C^{\infty}\left(C^{n}\right)$ satisfies $\operatorname{grad} \psi \neq 0$ on $\partial D$.

(1) Let $\mathscr{L}(\omega)$ denote the Levi form, i.e. the quadratic form

$$
\mathscr{L}(\omega) d z \overline{d z}=\left.\sum_{j, k} \frac{\partial^{2}(-\psi)}{\partial z_{j} \partial \bar{z}_{k}}\right|_{\omega} d z_{j} \overline{d z}_{k}
$$

restricted to the subspace $\left\{d z \in \boldsymbol{C}^{n}\left|\sum_{j}\left(\partial \psi / \partial z_{j}\right)\right|_{w} d z_{j}=0\right\}$ of $\boldsymbol{C}^{n}$.

(2) For $\omega_{1}, \omega_{2} \in D$, set $\rho\left(\omega_{1}, \omega_{2}\right)=\left|\omega_{1}-\omega_{2}\right|^{2}+\left|\left(\omega_{2}-\omega_{1}\right) \cdot(\partial \psi / \partial \omega)\right|_{\omega_{1}} \mid$. (See [2] again.)

(3) A smooth function $\varphi$ defined on $\bar{D} \times \bar{D}$ has weight $k$ (where $k \geqq 0$ is an integer or half-integer) if the following estimate holds.

$$
\left|\varphi\left(\omega_{1}, \omega_{2}\right)\right| \leqq C\left(\psi\left(\omega_{1}\right)+\psi\left(\omega_{2}\right)+\rho\left(\omega_{1}, \omega_{2}\right)\right)^{k}
$$

(4) Set

$$
\begin{aligned}
X(z, \omega)= & \psi(\omega)+\left.\sum_{j} \frac{\partial \psi}{\partial \omega_{j}}\right|_{\omega}\left(z_{j}-\omega_{j}\right) \\
& +\left.\frac{1}{2} \sum_{j, k} \frac{\partial^{2} \psi}{\partial \omega_{j} \partial \omega_{k}}\right|_{\omega}\left(z_{j}-\omega_{j}\right)\left(z_{k}-\omega_{k}\right) .
\end{aligned}
$$

AMS (MOS) subject classifications (1970). Primary 32F15, 32H10; Secondary $32 \mathrm{H} 15$.

Key words and phrases. Pseudoconvex domains, Bergman kernel function,. biholomorphic mapping, Levi form, Bergman metric, boundary behavior of mappings. 
Elementary calculations show that $X(z, \omega)$ has weight 1 , and that $|X(z, \omega)| \geqq c(\psi(z)+\psi(\omega)+\rho(z, \omega))$ in a region of the form $\boldsymbol{R}_{\delta}=$ $\{(z, \omega) \in \bar{D} \times \bar{D}|\psi(z)+\psi(\omega)+| z-\omega \mid<\delta\}$.

THEOREM 2. The Bergman kernel $K(z, \omega)$ for $D$ has an asymptotic expansion

$$
\begin{aligned}
K(z, \omega) \sim & c|\operatorname{grad} \psi(\omega)|^{2} \operatorname{det} \mathscr{L}(\omega) X^{-(n+1)}(z, \omega) \\
& +\sum_{j=1}^{\infty} \varphi_{j}(z, \omega) X^{-m_{j}}(z, \omega)+\tilde{\varphi}(z, \omega) \log X(z, \omega),
\end{aligned}
$$

where $c$ is a constant, $\varphi_{j}$ and $\tilde{\varphi}$ are smooth functions, "log" denotes the principal branch of the logarithm on $\{\operatorname{Re}(\zeta)>0\}$, weight $\left(\varphi_{j}\right)-m_{j} \geqq-n-\frac{1}{2}$, and weight $\left(\varphi_{j}\right)-m_{j} \rightarrow \infty$ as $j \rightarrow \infty$. The expansion (5) is valid in a region $R_{\delta}$, and the symbol " $\sim$ " means that for any integer $k$,

$$
\begin{aligned}
K(z, \omega) & -c|\operatorname{grad} \psi(\omega)|^{2} \operatorname{det} \mathscr{L}(\omega) X^{-(n+1)}(z, \omega) \\
& -\sum_{j=1}^{N} \varphi_{j}(z, \omega) X^{-m_{\jmath}}(z, \omega)-\tilde{\varphi}(z, \omega) \log X(z, \omega) \in C^{k}\left(\bar{R}_{\delta}\right)
\end{aligned}
$$

for $N$ large enough.

CoRollary. $K(z, z)=\Phi(z) \psi^{-(n+1)}(z)+\tilde{\Phi}(z) \log \psi(z)$, where $\Phi, \tilde{\Phi} \in$ $C^{\infty}(\bar{D})$ and $\Phi \neq 0$ near $\partial D$.

Although $\tilde{\Phi}$ vanishes on the unit ball, it can be nonzero, even on very smooth (say, real-analytic) domains.

The proof of Theorem 2 is based on an elementary fact.

Lemma 1. Given $p \in \partial D$, we can find a region $\tilde{D}$ internally tangent to $D$ to third order at $p$, and an explicit biholomorphic change of co-ordinates $F$ mapping a neighborhood of $p$ in $D$ to a neighborhood of $\widetilde{F}(p)$ in the unit ball.

Once Lemma 1 is established, we can use $\tilde{F}$ to pull the Bergman kernel from the unit ball back to $\tilde{D}$; and since $\tilde{D}$ so closely approximates $D$ near $p$, we may hope that the (known) Bergman kernel for $D$ provides a close approximation to the (unknown) Bergman kernel for $\tilde{D}$. Having thus obtained a candidate for an approximate Bergman kernel, we use a successive approximation procedure to prove (5).

Now we can attack Theorem 1 by using the corollary to Theorem 2 to make explicit differential-geometric calculations with the Bergman metric. We need two more definitions.

(6) For a fixed point $z^{0} \in D$ and a unit vector $\omega \in S^{2 n-1} \subseteq C^{n}$, let $t \rightarrow \gamma\left(t, \omega, z^{0}\right)$ be the path of a particle moving with unit speed (in the Bergman metric) along the geodesic in $D$ starting at $t=0$ at the point 
$z^{0}$ and travelling in the direction $\omega$. We say that $\left(z^{0}, \omega^{0}\right) \in D \times S^{2 n-1}$ is pseudotransversal if the map $\omega \rightarrow \pi_{z^{0}}(\omega)=\lim _{t \rightarrow \infty} \gamma\left(t, \omega, z^{0}\right)$ is well defined for $\omega$ close to $\omega^{0}$ in $S^{2 n-1}$ and provides a diffeomorphism of a small open neighborhood of $\omega^{0} \in S^{2 n-1}$ onto a small open neighborhood of $\pi_{z^{0}}(\omega) \in \partial D$.

(7) Let $t \rightarrow \gamma(t)$ be a geodesic in $D$, and define $\omega_{\gamma}(t)=$ the unit vector in the direction $d \gamma(t) / d t$. If $\left(\gamma(t), \omega_{\gamma}(t)\right) \in D \times S^{2 n-1}$ is pseudotransversal for all $t$ larger than some fixed $T$, then we call $\gamma$ a pseudotransversal geodesic.

LEMMA 2. (a) Every geodesic $\gamma(t)$ not remaining in a fixed compact subset of $D$ for all $t \geqq 0$ is pseudotransversal.

(b) Every point $p \in \partial D$ is $\pi_{z^{0}}\left(\omega^{0}\right)$ for a certain $\left(z^{0}, \omega^{0}\right) \in D \times S^{2 n-1}$.

Theorem 1 is a simple consequence of Lemma 2 and a result of Vormoor [1] which states that under the hypotheses of Theorem $1, F$ extends to a continuous mapping $\bar{F}: \bar{D}_{1} \rightarrow \bar{D}_{2}$. For, given $p_{1} \in \partial D_{1}$, we use Lemma 2(b) to find a geodesic $\gamma_{1}(t)$ in $D_{1}$ with $\lim _{t \rightarrow \infty} \gamma_{1}(t)=p_{1}$. Since $F$ is an isometry of Bergman metrics, the path $\gamma_{2}(t)=F\left(\gamma_{1}(t)\right)$ is a geodesic in $D_{2}$, and by Lemma 2(a), both $\gamma_{1}$ and $\gamma_{2}$ are pseudotransversal. Set $p_{2}=\lim _{t \rightarrow \infty} \gamma_{2}(t)$, and pick $T$ so large that $\left(z_{1}, \omega_{1}\right)=\left(\gamma_{1}(T), \omega_{\gamma_{1}}(T)\right)$ and $\left(z_{2}, \omega_{2}\right)=\left(\gamma_{2}(T), \omega_{\gamma_{2}}(T)\right)$ are both pseudotransversal. Since the differential of $F$ induces a diffeomorphism $(d F)^{\sim}$ between the unit tangent vectors based at $z_{1}$ and those based at $z_{2}$, we have a commutative diagram

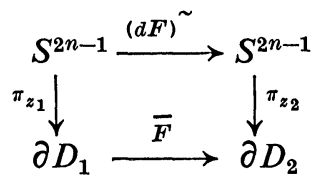

where the maps $\pi_{z_{1}}$ and $\pi_{z_{2}}$ are defined in small neighborhoods of $\omega_{1}=\pi_{z_{1}}^{-1}\left(p_{1}\right)$ and $\omega_{2}=\pi_{z_{2}}^{-1}\left(p_{2}\right)$. All the maps in the diagram, except $\bar{F}$, are already known to be diffeomorphisms. Hence $\bar{F}$ must also be a diffeomorphism from a neighborhood of $p_{1}$ to a neighborhood of $p_{2}$, which proves Theorem 1 .

\section{REFERENCES}

1. H. Grauert, Lecture given at a conference on several complex variables held in Paris, 1972.

2. E. M. Stein, Boundary behavior of holomorphic functions of several complex variables, Princeton Univ. Press, Princeton, N.J., 1972.

Department of Mathematics, University of Chicago, Chicago, Illinois 60637 